\title{
Pulmonary veins stenosis relief after an inappropriate radiofrequency catheter ablation of atrial fibrillation in a young non-competitive athlete
}

\author{
Berardo Sarubbi1, Gaetano Rea2, Giuseppe Santoro3, Enrico Melillo1, \\ Giancarlo Scognamiglio1, Maria Giovanna Russo3 \\ 1 Adult Congenital Heart Disease Unit, Monaldi Hospital, Naples \\ 2 Department of Radiology, Monaldi Hospital, Naples \\ 3 Pediatric Cardiology Unit, Second University of Naples, Monaldi Hospital, Naples, Italy
}

\begin{abstract}
One of the major complications of radiofrequency catheter ablation (RFCA) for atrial fibrillation (AF) is pulmonary vein stenosis (PVS). The natural history of PVS, especially when it involves more than one vein, leads to severe and irreversible pulmonary hypertension with end-stage right heart failure that can require, in extreme cases, even heart-lung transplantation.

We report the case of a young patient who underwent RFCA for a single lasting episode of AF and developed PVS years later. He was treated with ballon venoplasty followed by stent implantation in left pulmonary vein because of PVS relief. This reported case emphasizes the need of an adequate indication for RFCA for AF, considering the benefit-risk ratio especially in young patients with normal cardiac function.
\end{abstract}

Corresponding author: Dr. Berardo Sarubbi, Adult Congenital Heart Disease Unit, Monaldi Hospital, Via Leonardo Bianchi, 80131 Napoli, Italy. Tel. +39.081.7065288. E-mail: berardo.sarubbi@virgilio.it

Key words: Atrial fibrillation; catheter ablation; pulmonary veins stenosis; angioplasty.

Contributions: BS, manuscript writing and case supervision; GR, radiological images collection and description; GSa, catheter angiography images collection and description; GSc, EM, manuscript writing and data collection; MGR, case supervision.

Conflict of interest: The authors declare no conflict of interests and have not received any funding for this manuscript.

Acknowledgments: We thank all the medical and nursing staff for taking care of the patient.

Received for publication: 5 November 2017

Accepted for publication: 16 February 2018

(C) Copyright B. Sarubbi et al., 2018

Tipografia PI-ME Editrice, Italy

Monaldi Archives for Chest Disease 2018; 88:895

doi: 10.4081/monaldi.2018.895

This article is distributed under the terms of the Creative Commons Attribution Noncommercial License (by-nc 4.0) which permits any noncommercial use, distribution, and reproduction in any medium, provided the original author(s) and source are credited.

\section{Introduction}

Major complications of radiofrequency catheter ablation (RFCA) for atrial fibrillation (AF) are reported in up to $4.5 \%$ of cases [1], including death, cardiac tamponade, ischemic stroke and pulmonary vein stenosis (PVS) defined as the reduction of $>50 \%$ of pulmonary veins diameter [2].

The prevalence of PVS is estimated in up to $0.29 \%$ of cases [1]. However, real incidence remains a controversial issue, probably underestimated, because many patients are asymptomatic or quite symptomatic and there is not a routine assessing protocol for this condition. $\mathrm{Pa}$ tients with PVS may present with a huge variety of signs and symptoms like hemoptysis, dyspnea on exertion, chest pain, dry cough, fever, conditions often simulating other common pulmonary diseases like bronchopneumonia, interstitial lung disease and lung infarction on radiological and histopatological examination [3-4]. Since the clinical picture associated with PVS is extremely variable, a high index of suspicion is required. The gold standard for diagnosing PVS is widely accepted pulmonary venography, as angio-TC scan is considered less specific and sensitive. Severity of vein narrowing is defined according to the grade of luminal reduction in mild $(<50 \%)$, moderate $(50-70 \%)$ and severe $(>70 \%)$.

Currently there are not defined treatment strategies for PVS. Balloon venoplasty alone or with stent implanting is the principal treatment of pulmonary veins narrowing. It should be performed early, since late restoration of venous patency may not determine adequate perfusion of affected lungs segments, because of progressive periadventitial fibrosis and chronic vasoconstrinction of pulmonary vascular bed [5]. The rate of restenosis is quite high (up to 87\%) with balloon venoplasty alone, while is relatively lower after stenting (up to 57\%) [5]. A novel approach consists in implanting drug eluting stents with initial promising results obtained in a little number of patients [6]. Surgical treatment is an option reserved for complicated PVS, especially with left veins involvement.

\section{Case Report}

A 19-year-old apparently healthy man (a non-competitive basketball player) was admitted to our cardiac tertiary center because of progressive dyspnea on mild exertion and orthopnea. He denied chest pain and dizziness and referred frequent occurrence of fast irregular palpitations. Aged fifteen he underwent in a different hospital by an experienced team a RFCA through encircling pulmonary veins using a 3D electroanatomic mapping system for a single lasting episode of AF. After RFCA he underwent, over the time, repeated emergency admis- 
sions for further fast episodes of AF requiring pharmacological or electric treatment, despite antiarrhythmic prophylactic therapy (Sotalol, Flecainide or Propafenone). One year after the RFCA, he developed intermittent fever, with left sided chest pain, dry cough and dyspnea. A chest XR, at that time, showed suspicious infiltrates in subclavian and perihilar area of the left lung. His symptoms worsened over the years despite antibiotics and steroids.

At admission in our Hospital, ECG showed sinus rhythm with normal atrio-ventricular and intra-ventricular conduction and transthoracic echocardiography revealed normal size and systolic function of left and right ventricle, with a normal estimated pulmonary artery pressure. Six-minutes walking test revealed moderate impairment of functional capacity, with a 360 meters distance and a Borg index rating 6 . Chest XR showed patchy and small consolidations with disventilatory lesions in the medium field of the left lung. A multidetector CT angiogram (MDCT) was performed showing almost complete hypo-density of left pulmonary veins with severe stenosis of both veins. The left lung showed multiple nodular lesions, ground glass opacity area (GG0) and interstitial septal thickening (Figure 1). Considering the results of MDCT angiography, the previous history of RFCA for AF with encircling PV, recurrent AF episodes despite antiarrhythmic therapy, young age, and apparently normal morpho-functional echocardiographic findings, an iatrogenic pulmonary vein stenosis was suspected. The patient underwent cardiac catheterization under general anesthesia.

Right heart catheterization was performed through the right femoral vein, showing moderate increase of the pulmonary artery pressure (mean $20 \mathrm{mmHg}$ ) due to increased capillary vascular resistances. At pulmonary angiography, complete exclusion of the left pulmonary vascular tree was found, due to failure to drain of the left pulmonary veins. Thus, the attempt of recanalization of the occluded left pulmonary veins was planned. Following left atrial access was obtained by transseptal needle puncture (HeartSpan, Merit Medical Systems Inc., Malvern, PA, USA), a hand injection through a 4 Fr multipurpose catheter imaged a stump of the superior pulmonary vein that was carefully probed using a chronic occlusion coronary guide-wire (MiracleBros, Asahi Intecc, Aichi, Japan). After recanalization, the occluded pulmonary vein was progressively dilated using non-compliant coronary angioplasty balloons (NC Quantum Apex, Boston Scientific, Co., Marlborourgh, MA, USA) with good final result (Figure 2).

The clinical course was characterized by postoperative AF, treated with a $150 \mathrm{~J}$ biphasic electrical cardioversion (ECV). The patient was discharged on oral anticoagulant and Sotalol with a mild improvement in his symptoms.

Ten weeks later, in a routine outpatient visit, he referred worsening of his symptoms, with increasing dyspnea and reduced exercise capacity. He performed a further CT angiography showing absent visualization of both upper and inferior left pulmonary veins. For this reason, the patient was again admitted to our Division to perform a cardiac catheterization. Pulmonary angiography revealed normal caliber and course of pulmonary artery and absent visualization of left pulmonary veins, with normal flow in right pulmonary veins. The pulmonary artery pressure was $29 / 13 \mathrm{mmHg}$ (mean $17 \mathrm{mmHg}$ ). A pre-mounted $26 \mathrm{~mm}$ long peripheral stent (Lifestent Valeo, Bard Peripheral Vascular, Inc. Tempe, AZ, USA) was implanted and dilated to $8 \mathrm{~mm}$ (Figure 3). Final hand-injection angiography showed a freely patent pulmonary vein without vascular dissection and no pressure gradient across the implanted stent. The mean pulmonary artery pressure suddenly decreased to $15 \mathrm{mmHg}$ and a control pulmonary angiography showed a significant increase of the left pulmonary vascular tree opacification, albeit with a slow run-off of the contrast medium.

Immediately after cardiac catheterization, patient developed AF with a mean ventricular rate of $95 \mathrm{bpm}$, converted in sinus rhythm after ECV. The patient was discharged on oral anticoagulant, $100 \mathrm{mg} /$ daily aspirin, $50 \mathrm{mg}$ daily of Atenolol. During a two-years follow-up patient referred significant improvement of dyspnea and functional status, no more arrhythmic episodes, despite beta-blockers discontinuing. A new MDCT angiography was performed few months later and showed increased opacification and enhancement of the left pulmonary veins with patency of stent (Figure 3). His 6-minutes walking test distance was 560 meters with a Borg index rating 1 .

\section{Discussion}

In pediatric or adolescent subjects, $\mathrm{AF}$ is relatively rare, in the $\mathrm{ab}$ sence of either structural or functional heart disease, and in the absence of any identifiable hormonal or chemical cause. Although initial management should be conservative, in some cases during adolescence, the arrhythmia typically recurs multiple times despite medical therapy, creating a need for ablation therapy similar to the scenario in adults. The use of catheter ablation as been reported for paroxysmal supraventricular tachycardia and atrial fibrillation in young patients [7-8].
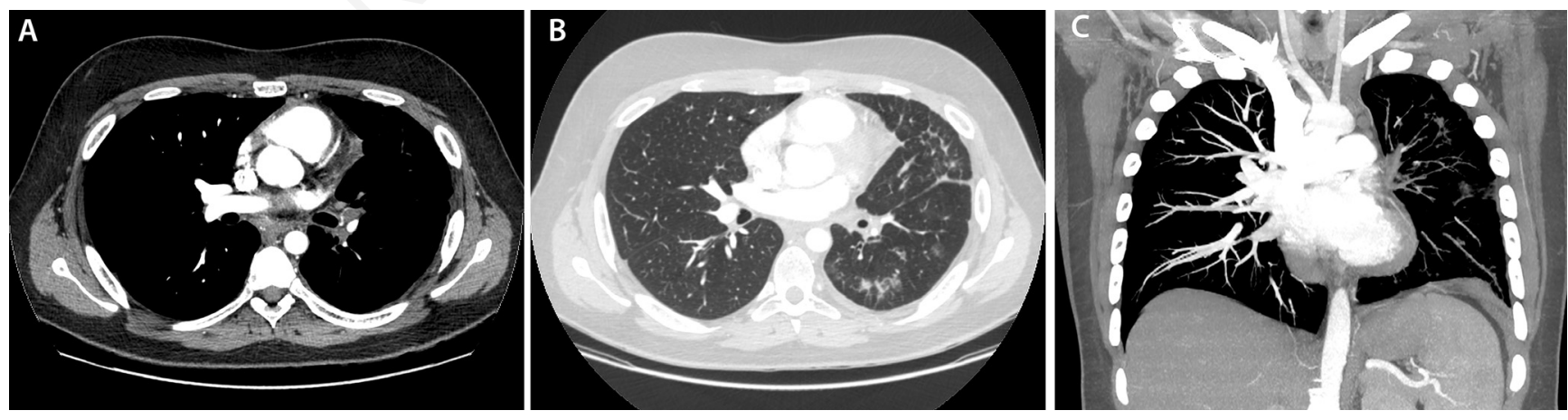

Figure 1. A) MDCT angiography showed almost complete hypodensity of left pulmonary veins with severe stenosis of both veins. Regular opacification of right interlobar artery and upper right pulmonary vein. B) MDCT angiography (lung window): multiple nodular lesions, GGO areas and linear parenchymal band in lingula and lower lobes. Interstitial septal thickening is prominent and also evident in subpleural region of lingula. These CT finding in absence of sign or symptoms of infectious disease are suggestive of pulmonary venous infarction related to venous stenosis. C) MDCT angiography, maximum intensity projection (MIP) 25 mm of thickness, coronal view: superb evaluation of lack of enhancement of pulmonary veins system to left. Regular opacification of pulmonary artery and veins to right. 
Specific technical details for ablation of either atrial flutter or fibrillation in the larger child are not particularly different from those in adults, however the decision of when to ablate can be quite different. After conversion from a first episode of atrial fibrillation, it is not clear the necessity of a prophylactic antiarrhythmic drug. After recurrences, the threshold for ablation can be similar to that in adults. The use of ablation therapy for the rare cases of $\mathrm{AF}$ in pediatric patients is also appealing, but the high emphasis on safety over efficacy for all children mandates that a decision to use RFCA in this age group be considered only after failure of multiple antiarrhythmic agents. Furthermore, the technique chosen should be the safest and most effective because complications such as pulmonary vein stenosis can be devastating to a child.

In this setting, it is important to note that as the techniques used for catheter ablation of AF are continuing to evolve rapidly, cryoablation could expect to produce minimal PVS due to the lack of endothelium disruption and lack of hyperthermic injury with this procedure [9], even if the RFCA can be made without or with minimally radiation exposure.
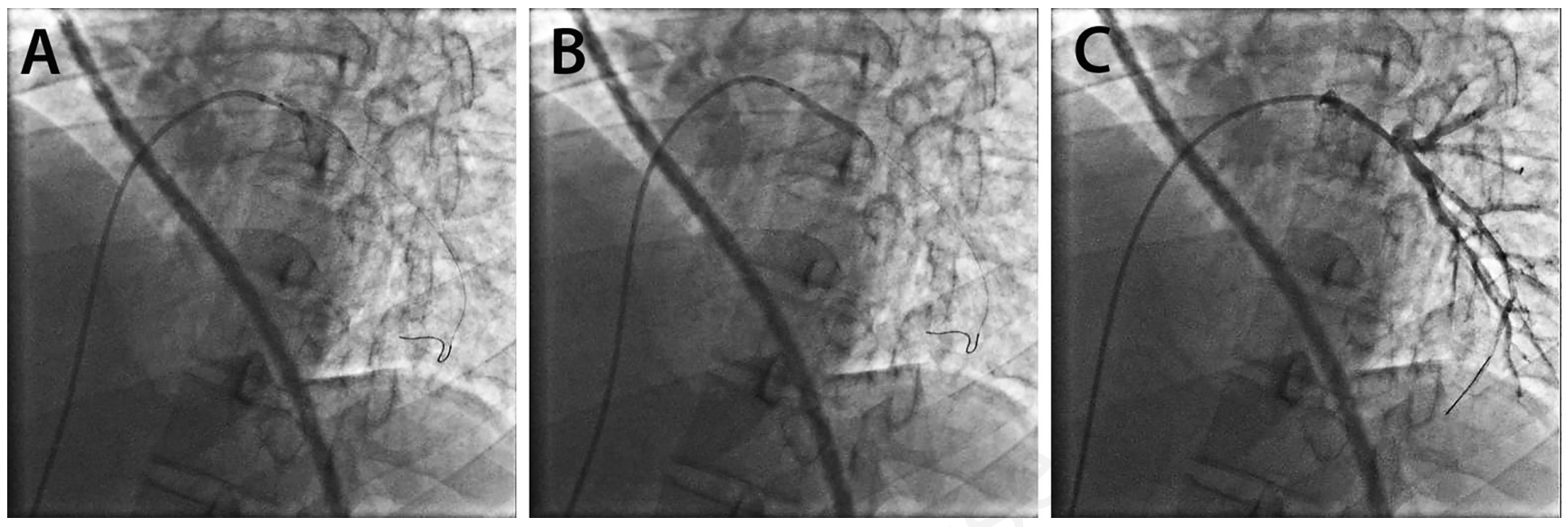

Figure 2. A) Right heart catheterization showing occluded left pulmonary vein. B) Balloon angioplasty of occluded left pulmonary vein. C) Control pulmonary angiography after balloon angioplasty showing significant increase of left pulmonary vascular tree.
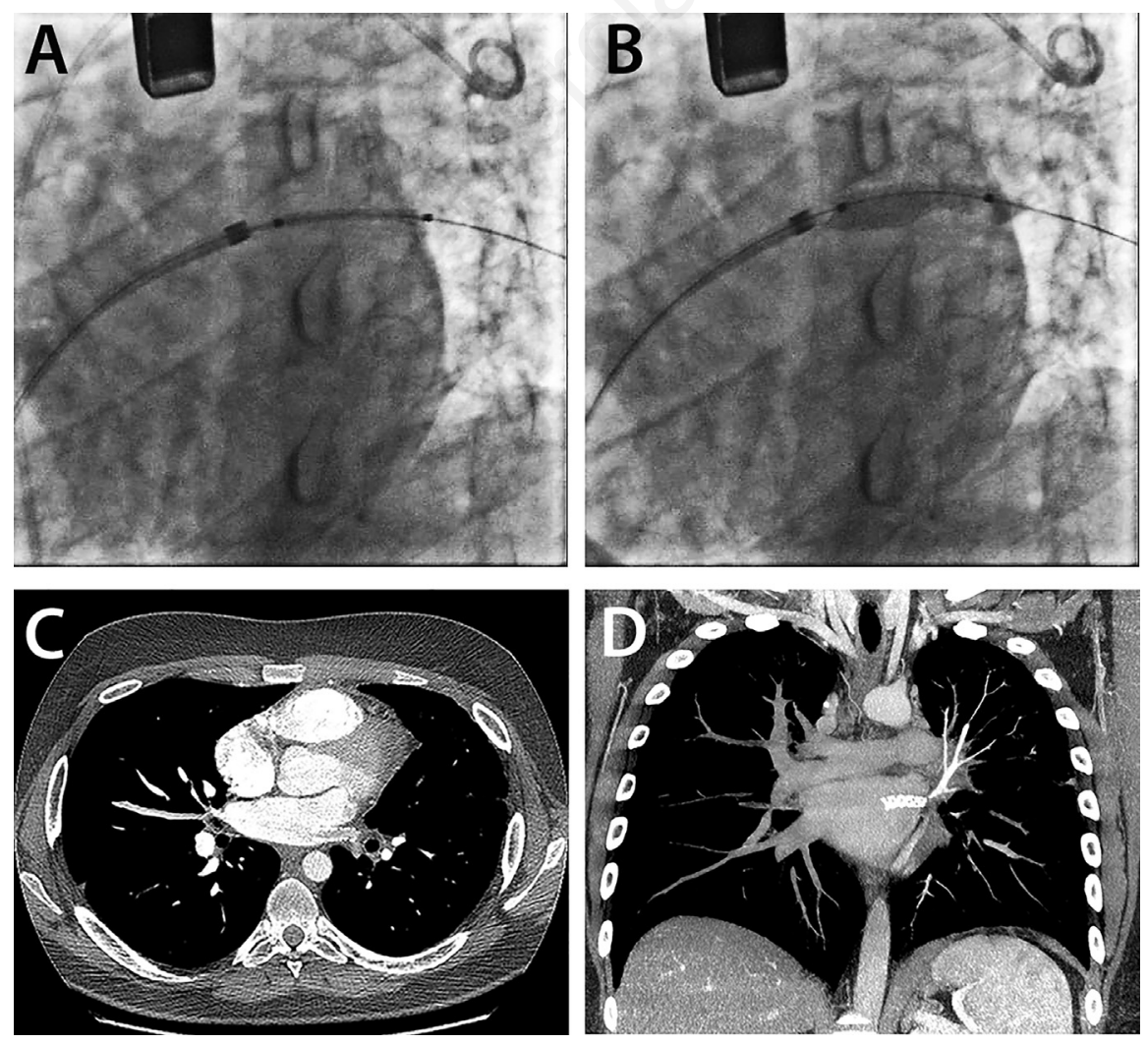

Figure 3. A,B) Angiogram showing stenting of left pulmonary vein C) MDCT angiography post treatment: clear evidence of stent in common left venous vessel to left and moderate opacification inside in a venous contrast phase. D) MDCT angiography, maximum intensity projection (MIP) $15 \mathrm{~mm}$ of thickness, coronal view in late venous phase: device (stent) in common left venous pulmonary veins with increase of opacification of the left upper pulmonary vein and mild enhancement also related to lower left pulmonary vein. 
In the reported case, RFCA of AF through encircling pulmonary veins for a single lasting episode of AF has to be considered an inappropriate, too aggressive, procedure in a fifteen-healthy subject who not already experimented previous antiarrhythmic treatment, even if the benefit/risk ratio could be considered higher in such young patient with lone AF than in presence with other cardiopathy.

Clinical and instrumental tools made suspicion of PVS. A MDCT gave enough data related to the pulmonary impairment prior to perform cardiac catheterization for pulmonary venography.

In the reported case, balloon venoplasty with stent implanting was considered the only possibility to relief the PVS as surgical treatment was an option not readily and easily performed and an earlier relief was urgently required to avoid an irreversible pulmonary hypertension [10].

Finally, it needs to be defined the possible correlation between PVS and $\mathrm{AF}$ recurrences as the patient showed repeated episodes of arrhythmias in the presence of recognized veins obstruction and lack of recurrences during the two years follow-up after PVS relief. A possible mechano-electrical feedback could explain the mechanism of AF recurrences in this case [11].

\section{Conclusions}

PVS is a rare but insidious condition and clinicians should be aware in patients who underwent ablation procedure for AF, especially in young patients without a structural or functional heart disease with a not established risk of recurrences.

\section{References}

1. Cappato R, Calkins H, Chen SA, et al. Updated worldwide survey on the methods, efficacy, and safety of catheter ablation for human atrial fibrillation. Circ Arrhythm Electrophysiol 2010;3:32-8.
2. De Greef Y, Tavernier R, Raeymaeckers S, et al. Prevalence, characteristics, and predictors of pulmonary vein narrowing after isolation using the primary vein ablation catheter. Circ Arrhythm Electrophysiol 2012;5:52-60.

3. Holmes DR, Monahan KH, Packer D. Pulmonary vein stenosis complicating ablation for atrial fibrillation: clinical spectrum and interventional considerations. JACC Cardiovasc Interv 2009;2:267-76.

4. Kwon MR, Lee HY, Cho JH, Um SW. Lung infarction due to pulmonary vein stenosis after ablation therapy for atrial fibrillation misdiagnosed as organizing pneumonia: sequential changes on CT in two cases. Korean J Radiol 2015;16:942-6.

5. Rostamian A, Narayan SM, Thomson L, et al. The incidence, diagnosis, and management of pulmonary vein stenosis as a complication of atrial fibrillation ablation. J Interv Card Electrophysiol 2014;40:63-74.

6. De Potter TJ, Schmidt B, Chun KR, et al. Drug-eluting stents for the treatment of pulmonary vein stenosis after atrial fibrillation ablation. Europace 2011;13:57-61.

7. Kugler JD, Danford DA, Houston K, Felix G. Radiofrequency catheter ablation for paroxysmal supraventricular tachycardia in children and adolescents without structural heart disease. Pediatric EP Society, Radiofrequency Catheter Ablation Registry. Am J Cardiol 1997;80:1438-43.

8. Nanthakumar K., Lau YR, Plumb VJ, et al. Electrophysiological findings in adolescents with atrial fibrillation who have structurally normal hearts. Circulation 2004;110:117-23.

9. Matsuda J, Miyazaki S, Nakamura $\mathrm{H}$, et al. Pulmonary vein stenosis after second-generation cryoballoon ablation. J Cardiovasc Electrophysiol 2017;28:298-303.

10. Verma I, Tripathi H, Sikachi RR, Agrawal A. Pulmonary hypertension due to radiofrequency catheter ablation (RFCA) for atrial fibrillation: The lungs, the atrium or the ventricle? Heart Lung Circ 2016;25:1177-83.

11. Sarubbi B, Ducceschi V, Santangelo L, Iacono A. Arrhythmias in patients with mechanical ventricular dysfunction and myocardial stretch: role of mechanoelectrical feedback. Can J Cardiol 1998;14:245-52. 